\title{
Self-Packing of Centrally Symmetric Convex Bodies in $\mathbb{R}^{2}$
}

\author{
P. G. Doyle,${ }^{1}$ J. C. Lagarias, ${ }^{1}$ and D. Randall ${ }^{2}$ \\ ${ }^{1}$ AT\&T Bell Laboratories, Murray Hill, NJ 07974, USA \\ ${ }^{2}$ University of California at Berkeley, Berkeley, CA 94720 , USA
}

\begin{abstract}
Let $B$ be a compact convex body symmetric around 0 in $\mathbb{R}^{2}$ which has nonempty interior, i.e., the unit ball of a two-dimensional Minkowski space. The self-packing radius $\rho(m, \mathbf{B})$ is the smallest $t$ such that $t \mathbf{B}$ can be packed with $m$ translates of the interior of $\mathbf{B}$. For $m \leq 6$ we show that the self-packing radius $\rho(m, \mathbf{B})=1+2 / \alpha(m, \mathbf{B})$ where $\alpha(m, \mathbf{B})$ is the Minkowski length of the side of the largest equilateral $m$-gon inscribed in $\mathbf{B}$ (measured in the Minkowski metric determined by B). We show $\rho(6, B)=\rho(7, B)=3$ for all $B$, and determine most of the largest and smallest values of $\rho(m, \mathbf{B})$ for $m \leq 7$. For all $m$ we have
\end{abstract}

$$
\left(\frac{m}{\delta(\mathbf{B})}\right)^{1 / 2}-\frac{3}{2} \leq \rho(m, \mathbf{B}) \leq\left(\frac{m}{\delta(\mathbf{B})}\right)^{1 / 2}+1
$$

where $\delta(\mathbf{B})$ is the packing density of $\mathbf{B}$ in $\mathbb{R}^{2}$.

\section{Introduction}

A compact convex body $\mathbf{B}$ symmetric about $\mathbf{0}$ in $\mathbb{R}^{2}$ which is the closure of its interior is the unit ball of a two-dimensional Banach space, and conversely. A finite-dimensional Banach space is also called a Minkowski space, which is the terminology we shall use here, and we call any such B a Minkowski disk. This paper studies the packing of translates of a Minkowski disk $\mathbf{B}$ into a homothetic copy $t \mathbf{B}$ of such a disk, where $t>1$. By a packing we mean that the interiors of the translated bodies are mutually disjoint; their boundaries are permitted to overlap. The self-packing radius $\rho(m, \mathbf{B})$ is the minimal radius $t$ such that $m$ translates of $\mathbf{B}$ can be packed in $t \mathbf{B}$. We study the problem of determining $\rho(m, \mathbf{B})$, and call this the self-packing problem.

The self-packing problem is actually a problem in two-dimensional affine 
geometry, since the notions of translation, homothety, and packing are all affine invariants. It turns out, however, that the Minkowski metric $d_{\mathbf{B}}(\cdot, \cdot)$ having $\mathbf{B}$ as its unit ball plays a useful role in studying this problem.

Our main results are as follows. For small values of $m$ we show that the self-packing radius is related to equilateral Minkowski $m$-gons inscribed in the Minkowski disk B. Such equilateral polygons exist having a vertex at an arbitrary boundary point of $\mathbf{B}$, and we define the quantity $\alpha(m, \mathbf{B})$ to the maximum Minkowski side-length of an equilateral Minkowski $m$-gon inscribed in $\mathbf{B}$. In Section 3 we show that, for $m \leq 6$,

$$
\rho(m, \mathbf{B})=1+\frac{2}{\alpha(m, \mathbf{B})}
$$

That is, there always exists an extremal configuration with the centers of the packed disks located at the vertices of an equilateral Minkowski $m$-gon. We deduce among other results that $\rho(6, \mathbf{B})=\rho(7, \mathbf{B})=3$ for all Minkowski disks B. For large values of $m$ the self-packing radius is obviously related to the packing density $\delta(\mathbf{B})$ of $\mathbb{R}^{2}$ by copies of $B$. In Section 4 we prove that in fact

$$
\left(\frac{m}{\delta(\mathbf{B})}\right)^{1 / 2}-\frac{3}{2} \leq \rho(m, \mathbf{B}) \leq\left(\frac{m}{\delta(\mathbf{B})}\right)^{1 / 2}+1
$$

Our study of these questions was motivated by a conjecture of Du et al. [3] that for an arbitrary Minkowski metric in $\mathbb{R}^{2}$ there is a finite point set such that the Steiner ratio ST/MST $\leq \sqrt{3} / 2$, where MST is the length of the minimal spanning tree and ST is the length of the Steiner minimal tree for that metric. This conjecture cannot be strengthened, since Du and Hwang [4] recently proved that for the Euclidean metric all finite point sets have ST/MST $\geq \sqrt{3} / 2$. Theorem 3.4 below gives a relation between the Steiner ratio and the self-packing problem.

\section{Equilateral Minkowski Polygons}

Let $\mathbf{B}$ denote a Minkowski disk and let $d(\mathbf{x}, \mathbf{y})$ (sometimes $d_{\mathbf{B}}(\mathbf{x}, \mathbf{y})$ if $\mathbf{B}$ varies) denote its associated distance function, which satisfies the triangle inequality

$$
d(\mathbf{x}, \mathbf{y}) \leq d(\mathbf{x}, \mathbf{z})+d(\mathbf{z}, \mathbf{y})
$$

We derive some properties of such distance functions, and use them to prove the existence of equilateral Minkowski polygons inscribed in B having a vertex at an arbitrary boundary point.

Lemma 2.0. For any triangle $\mathrm{xyz}$ in $\mathbb{R}^{2}$ and any point $\mathrm{w}$ on the segment $\mathbf{y z}$,

$$
d(\mathbf{x}, \mathbf{w}) \leq \max (d(\mathbf{x}, \mathbf{y}), d(\mathbf{x}, \mathbf{z})) .
$$


Proof. Translate the triangle so that $\mathbf{x}$ is the center of the Minkowski unit ball. Then $\mathbf{y}$ and $\mathbf{z}$ are contained in the Minkowski ball $\lambda \mathbf{B}$ where $\lambda=\max (d(\mathbf{x}, \mathbf{y})$, $d(\mathbf{x}, \mathbf{z})$ ). By convexity $w$ is contained in $\lambda \mathbf{B}$ so (2.1) follows.

Remark. This result also follows from the fact, true in any normed space, that the distance function $d(\mathbf{x}, \mathbf{y})$ is a convex function of $\mathbf{y}$ :

$$
\begin{aligned}
d\left(\mathbf{0},(1-t) \mathbf{y}_{0}+t \mathbf{y}_{1}\right) & =\left\|(1-t) \mathbf{y}_{0}+t \mathbf{y}_{1}\right\| \\
& \leq|1-t|\left\|\mathbf{y}_{0}\right\|+|t|\left\|\mathbf{y}_{1}\right\| \\
& =t d\left(0, \mathbf{y}_{0}\right)+(1-t) d\left(0, \mathbf{y}_{1}\right)
\end{aligned}
$$

as long as $0 \leq t \leq 1$.

Lemma 2.1. If $\mathbf{x}, \mathbf{y}$ are two points in $\mathbf{B}$ with $d(\mathbf{x}, \mathbf{y})>1$ and $\mathbf{x}^{\prime}, \mathbf{y}^{\prime}$ are the radial projections of $\mathbf{x}, \mathbf{y}$ onto the boundary of $\mathbf{B}$, then

$$
d\left(\mathbf{x}^{\prime}, \mathbf{y}^{\prime}\right) \geq d(\mathbf{x}, \mathbf{y})
$$

Proof. Since $d(\mathbf{x}, \mathbf{y})>1$ neither point can be the center $\mathbf{0}$ so the radial projections are well defined. If $\mathbf{0}$ is on the line determined by $\mathbf{x y}$, then $\mathbf{0}$ must lie between $\mathbf{x}^{\prime}$ and $\mathbf{y}$ so $\mathbf{x}^{\prime} \mathbf{y}^{\prime}$ is a diameter and (2.2) holds. If $\mathbf{0}$ is not on $\mathbf{x y}$, then by moving the line determined by $\mathbf{x y}$ parallel to itself away from $\mathbf{0}$ until it hits an endpoint $\mathbf{x}^{\prime}$ or $y^{\prime}$ (say $x^{\prime}$ ), we obtain a new segment $x^{\prime} y^{\prime \prime}$ parallel to $x y$ (see Fig. 2.1) and by similar triangles

$$
d\left(\mathbf{x}^{\prime}, \mathbf{y}^{\prime \prime}\right) \geq d(\mathbf{x}, \mathbf{y})>1
$$

Now by $(2.1)$

$$
\begin{aligned}
d\left(\mathbf{x}^{\prime}, \mathbf{y}^{\prime \prime}\right) & \leq \max \left(d\left(\mathbf{0}, \mathbf{x}^{\prime}\right), d\left(\mathbf{x}^{\prime}, \mathbf{y}^{\prime}\right)\right) \\
& =\max \left(1, d\left(\mathbf{x}^{\prime}, \mathbf{y}^{\prime}\right)\right)
\end{aligned}
$$

With (2.3) this yields

$$
d\left(\mathbf{x}^{\prime}, \mathbf{y}^{\prime \prime}\right) \leq d\left(\mathbf{x}^{\prime}, \mathbf{y}^{\prime}\right)
$$

and (2.2) follows from (2.3) and (2.4).

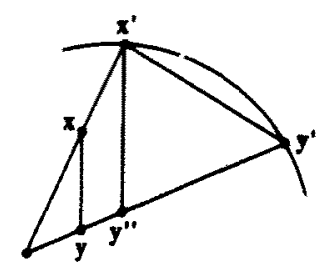

Fig. 2.1. Radially projecting $x, y$ to obtain $x^{\prime}, y^{\prime}$. 
Remarks. (1) The hypothesis $d(\mathbf{x}, \mathbf{y})>1$ is necessary to conclude (2.2), for otherwise we can take $\mathbf{x}, \mathbf{y}$ on a radial segment so that $\mathbf{x}^{\prime}=\mathbf{y}^{\prime}$.

(2) The argument for (2.1) shows that if $\mathbf{B}$ is strictly convex and $\mathbf{w}$ is strictly interior to the segment $\mathbf{x y}$, then

$$
d(\mathbf{x}, \mathbf{w})<\max (d(\mathbf{x}, \mathbf{y}), d(\mathbf{y}, \mathbf{z}))
$$

(3) Lemma 2.1 comes down to the fact that if $\|\mathbf{x}\|=\|\mathbf{y}\|=1$ and $0<t<1$, then $\|\mathbf{x}-\mathbf{y}\| \geq\|\mathbf{x}-t \mathbf{y}\|$ whenever $\|\mathbf{x}-t \mathbf{y}\| \geq 1$. To see this, note that

$$
\begin{aligned}
\|\mathbf{x}-t \mathbf{y}\| & =\|(1-t) \mathbf{x}+t(\mathbf{x}-\mathbf{y})\| \\
& \leq(1-t)\|\mathbf{x}\|+t\|\mathbf{x}-\mathbf{y}\| \\
& =1+t(\|\mathbf{x}-\mathbf{y}\|-1)
\end{aligned}
$$

In order to have $\|\mathbf{x}-t \mathbf{y}\| \geq 1$ the slope $\|\mathbf{x}-\mathbf{y}\|-1$ in this linear estimate must be nonnegative. Then

$$
\begin{aligned}
\|\mathbf{x}-t \mathbf{y}\| & \leq 1+t(\|\mathbf{x}-\mathbf{y}\|-1) \\
& \leq 1+\|\mathbf{x}-\mathbf{y}\|-1=\|\mathbf{x}-\mathbf{y}\|
\end{aligned}
$$

Lemma 2.2. Let $\mathbf{w}, \mathbf{x}, \mathbf{y}, \mathbf{z}$ be four clockwise ordered points on the boundary of the Minkowski unit ball $\mathbf{B}$ such that the line determined by $\mathbf{w z}$ separates $\mathbf{x}$ and $\mathbf{y}$ from the origin $\mathbf{0}$. Here $\mathbf{w}=\mathbf{x}$ and $\mathbf{y}=\mathbf{z}$ are allowed. Then

$$
d(\mathbf{w}, \mathbf{z}) \geq d(\mathbf{x}, \mathbf{y})
$$

If $\mathbf{B}$ is strictly convex and (w, $\mathbf{z})$ does not coincide with $(\mathbf{x}, \mathbf{y})$, then

$$
d(\mathbf{w}, \mathbf{z})>d(\mathbf{x}, \mathbf{y})
$$

Proof. The configuration is pictured in Fig. 2.2. Move the line determined by xy parallel to itself toward 0 until it hits the segment wz. Without loss of generality

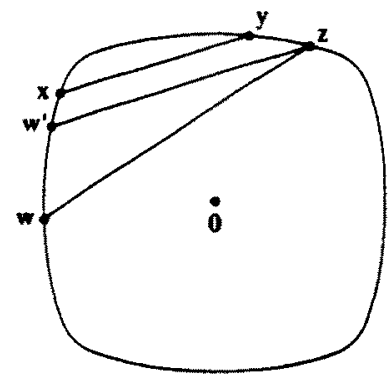

Fig. 2.2. Comparing chords $w z$ and $x y$. 


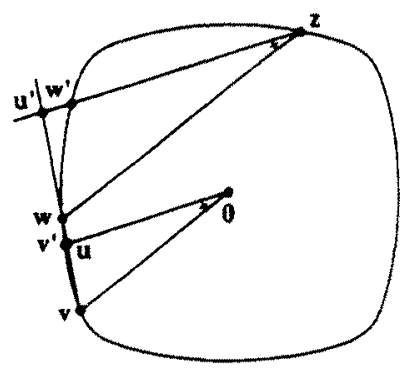

Fig. 2.3. Case 1 of Lemma 2.2.

it hits $\mathbf{z}$ and we obtain a segment $\mathbf{w}^{\prime} \mathbf{z}$ with $\mathbf{w}^{\prime}$ separated from 0 by the line $\mathbf{w z}$. Also

$$
d\left(\mathbf{w}^{\prime}, \mathbf{z}\right) \geq d(\mathbf{x}, \mathbf{y})
$$

by the convexity of $\mathbf{B}$, and the fact that the line wz separates $\mathbf{x}$ and $\mathbf{y}$ from $\mathbf{0}$. For the first part it suffices to show

$$
d(\mathbf{w}, \mathbf{z}) \geq d\left(\mathbf{w}^{\prime}, \mathbf{z}\right)
$$

Let $0 \mathbf{v}^{\prime}$ be a radius of $\mathbf{B}$ parallel to the (oriented) line $\mathbf{z w}^{\prime}$ and let $0 \mathrm{v}$ be parallel to the oriented line $\mathbf{w z}$.

Case 1: $\mathbf{v}^{\prime}$ lies between $\mathbf{w}$ and $\mathbf{v}$ on the boundary of $\mathbf{B}$ (Fig. 2.3). If so, the chord $w v$ intersects the radius $0 v$, and call the intersection point $u$. Next let $u^{\prime}$ be the intersection of the line determined by $\mathbf{z w ^ { \prime }}$ with the line determined by wv. This point exists since $\mathbf{w v}$ is not parallel to $0 v^{\prime}$, and by convexity of $B$ the point $\mathbf{w}^{\prime}$ separates $\mathbf{u}^{\prime}$ and $\mathbf{z}$, and $\mathbf{u}^{\prime}$ lies outside (or on the boundary) of $\mathbf{B}$. Now

$$
\frac{d(\mathbf{z}, \mathbf{w})}{d\left(\mathbf{z}, \mathbf{w}^{\prime}\right)} \geq \frac{d(\mathbf{z}, \mathbf{w})}{d\left(\mathbf{z}, \mathbf{u}^{\prime}\right)}=\frac{d(\mathbf{0}, \mathbf{v})}{d(\mathbf{0}, \mathbf{u})} \geq \frac{d(\mathbf{0}, \mathbf{v})}{d\left(\mathbf{0}, \mathbf{v}^{\prime}\right)}=1,
$$

where the inner equality uses similarity of triangles zu'w and Ouv. This proves (2.7) in this case.

Case 2: $\mathbf{w}$ lies between $\mathbf{v}^{\prime}$ and $\mathbf{v}$ on the boundary of $\mathbf{B}$ (Fig. 2.4). If so the chord $\mathbf{v}^{\mathbf{v}} \mathbf{v}$ intersects the chord $\mathbf{w z}$ in a point $\mathbf{u}$, and the extended line $\mathbf{v}^{\prime} \mathbf{v}$ intersects the line $\mathbf{z w}^{\prime}$ in a point $\mathbf{u}^{\prime}$, with $\mathbf{w}$ separating $\mathbf{u}^{\prime}$ from $\mathbf{z}$. Now

$$
\frac{d(\mathbf{z}, \mathbf{w})}{d\left(\mathbf{z}, \mathbf{w}^{\prime}\right)} \geq \frac{d(\mathbf{z}, \mathbf{w})}{d\left(\mathbf{z}, \mathbf{u}^{\prime}\right)} \geq \frac{d(\mathbf{z}, \mathbf{u})}{d\left(\mathbf{z}, \mathbf{u}^{\prime}\right)}=\frac{d\left(\mathbf{0}, \mathbf{v}^{\prime}\right)}{d(\mathbf{0}, \mathbf{v})}=1,
$$

where similarity of triangles zu'u and $0 v^{\prime} v$ was used. This proves $(2.7)$ in this case.

These cases are exhaustive, so (2.7) follows.

It remains to prove strict inequality when $B$ is strictly convex. Now if $z$ is the 


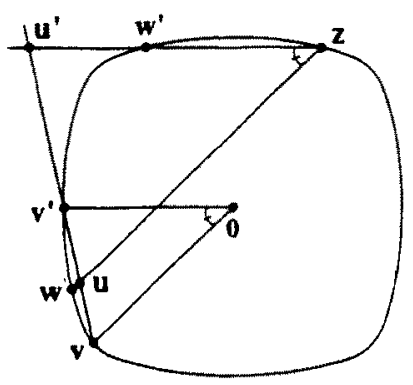

Fig. 2.4. Case 2 of Lemma 2.2 .

endpoint of wz first hit on moving the line determined by $\mathbf{x y}$ parallel to itself, and if $\mathbf{y} \neq \mathbf{z}$, then $d\left(\mathbf{w}^{\prime}, \mathbf{z}\right)>d(\mathbf{x}, \mathbf{y})$ in (2.6) and so $d(\mathbf{w}, \mathbf{z})>d(\mathbf{x}, \mathbf{y})$. Now suppose $\mathbf{y}=\mathbf{z}$, while $\mathbf{x}=\mathbf{w}^{\prime} \neq \mathbf{w}$. Then in Case 1 we have $d\left(\mathbf{z}, \mathbf{u}^{\prime}\right)>d\left(\mathbf{z}, \mathbf{w}^{\prime}\right)$ by $(2.5)$, and so strict inequality holds in (2.8). Similarly in Case $2 d\left(\mathbf{z}^{\prime}, \mathbf{u}^{\prime}\right)>d\left(\mathbf{z}, \mathbf{w}^{\prime}\right)$ and strict inequality holds in (2.9). Thus strict inequality holds in (2.7) and the second part of Lemma 2.2 follows.

Lemma 2.2 has as an immediate consequence:

Lemma 2.3. For a fixed point $\mathbf{x}$ on the boundary of $\mathbf{B}$, the quantity $d(\mathbf{x}, \mathbf{y})$ is nondecreasing as $\mathbf{y}$ moves clockwise (resp. counterclockwise) on the boundary of $\mathbf{B}$ starting at $\mathbf{X}$ until it reaches the point $\mathbf{X}^{\prime}$ antipodal to $\mathbf{x}$. It is strictly increasing if $\mathbf{B}$ is strictly convex.

Proof. If $\mathbf{y}$ and $\mathbf{y}^{\prime}$ are two such points with clockwise ordering $\mathbf{x}, \mathbf{y}, \mathbf{y}^{\prime}, \mathbf{x}^{\prime}$, then the line determined by $\mathbf{x y}^{\prime}$ separates $\mathbf{y}$ from $\mathbf{0}$. Lemma 2.2 then yields

$$
d(\mathbf{x}, \mathbf{y}) \geq d\left(\mathbf{x}, \mathbf{y}^{\prime}\right)
$$

as well as strict inequality in the case of strict convexity of $\mathbf{B}$.

Lemma 2.4. For any $m \geq 3$ and any point $\mathrm{x}$ on the boundary of a Minkowski disk $\mathbf{B}$ there exists a convex $m$-gon inscribed in $\mathbf{B}$ having $\mathbf{x}$ as a vertex which is equilateral in the Minkowski metric $d_{\mathbf{B}}$. If $\mathbf{B}$ is strictly convex, this $m$-gon is unique. For all $\mathbf{B}$ the Minkowski edge length of all convex equilateral m-gons through $\mathbf{x}$ is unique.

We remark that most bodies B possess two convex equilateral Minkowski $m$-gons having perimeters of different Minkowski lengths.

Proof. Let $\partial \mathbf{B}$ denote the boundary of B. For each $\alpha$ with $0 \leq \alpha \leq 2$ define the function $T_{\alpha}: \partial \mathbf{B} \rightarrow \partial \mathbf{B}$ which assigns to each point $\mathbf{x} \in \partial \mathbf{B}$ the first point $\mathbf{y} \in \partial \mathbf{B}$ with $d(\mathbf{x}, \mathbf{y})=\alpha$ which is encountered moving counterclockwise around $\partial \mathbf{B}$ from $\mathbf{x}$. (Note that $T_{0}$ is the identity map.) The continuity of $d(x, \cdot)$ and Lemma 2.3 guarantee that for $\alpha<2$ the set of points on $\partial \mathbf{B}$ with $d(\mathbf{x}, \mathbf{y})=\alpha$ form two disjoint 
closed arcs on $\partial \mathbf{B}$ separated by $\mathbf{x}$ and $-\mathbf{x}$, while for $d(\mathbf{x}, \mathbf{y})=2$ the set is a single closed arc containing $-\mathbf{x}$. Furthermore, if $\mathbf{B}$ is strictly convex these closed arcs are necessarily points. These facts guarantee that $T_{\alpha}$ is defined. We also associate to $T_{\alpha}(\mathbf{x})$ a rotation measure $\theta(\mathbf{x}, \alpha)$ satisfying $0 \leq \theta(\mathbf{x}, \alpha) \leq \pi$ which is defined by $\theta(\mathbf{x}, \alpha) \equiv \varphi_{1}-\varphi_{2}(\bmod \pi)$ where $\mathbf{x}=r_{1} e^{i \varphi_{1}}$ and $T_{\alpha}(\mathbf{x})=r_{2} e^{i \varphi_{2}}$ are viewed as complex numbers.

Now define $T:[0,2] \times \partial \mathbf{B} \rightarrow \partial \mathbf{B}$ by $T(\alpha, \mathbf{x}):=T_{\alpha}(\mathbf{x})$. We claim that if $\mathbf{B}$ is strictly convex, then $T$ is continuous in both variables. This follows from the continuity of $d(\mathbf{x}, \cdot)$ and Lemma 2.3. If $\mathbf{B}$ is not strictly convex, then $T$ may fail to be continuous in either variable separately.

Suppose now that $\mathbf{B}$ is strictly convex. The claim above implies that the rotation measure $\theta:[0,2] \times \partial \mathbf{B} \rightarrow[0, \pi]$ is continuous in both variables, and by Lemma 2.3 it is a strictly increasing function of $\alpha$ for each fixed $\alpha \in \partial \mathbf{B}$. Define a rotation measure for the iterated map $T_{\alpha}^{(m)}$ by

$$
\theta^{(m)}(\mathbf{x}, \alpha):=\sum_{i=0}^{m-1} \theta\left(T^{(i)}(\mathbf{x}), \alpha\right) .
$$

We seek the minimal $\alpha>0$ yielding a fixed point $T_{\alpha}^{(m)}(\mathbf{x})=\mathbf{x}$. This corresponds to $m$ iterations of $T_{\alpha}$ rotating around the boundary exactly once, i.e., to a solution to

$$
\theta^{(m)}(\mathbf{x}, \alpha)=2 \pi .
$$

Lemmas 2.2 and 2.3 together guarantee that $\theta^{(m)}:[0,2] \times \partial \mathbf{B} \rightarrow[0, m \pi]$ is continuous in both variables, and strictly increasing in $\alpha$ for fixed $\mathbf{x}$. Also the strict convexity of $\mathbf{B}$ forces $\theta(\mathbf{x}, 2)=\pi$ hence $\theta^{(m)}(\mathbf{x}, 2)=m \pi$. Thus there is a unique value of $\alpha$ solving (2.11), and associated to it is a unique equilateral Minkowski $m$-gon having $\mathbf{x}$ as a vertex.

We treat the case of arbitrary convex $B$ by a limiting process. Inscribe inside $\mathbf{B}$ a circular disk $\mathbf{D}_{R}$ of radius $R$, say, and form the sequence of bodies $\mathbf{B}_{t}=$ $t \mathbf{B}+(1-t) \mathbf{D}_{R}$ for $0 \leq t \leq 1$. Then $\mathbf{B}_{t}$ is strictly convex for $0 \leq t<1$, and it has a unique inscribed equilateral Minkowski $m$-gon of Minkowski length $\alpha(t)$ having $\mathbf{x}(t):=(t+(1-t) R /\|\mathbf{x}\|) \mathbf{x}$ as a vertex. Since $\mathbf{B}$ is compact we can extract a convergent subsequence of $m$-gons converging to a limit $m$-gon inscribed in $\partial \mathbf{B}$. By continuity of the Minkowski metric $d_{\mathbf{B}}$ as $\mathbf{B}$ is continuously deformed, this limit polygon is equilateral in the Minkowski metric of $\mathbf{B}$. This proves existence.

In the case of nonstrictly convex $\mathbf{B}$ an equilateral $m$-gon through a point $\mathbf{x}$ need not be unique. (The square $\mathbf{S}$ has infinitely many equilateral Minkowski triangles of side length 2 having two vertices at corners of $\mathbf{S}$.) However, its length is unique. This may be seen as follows. The functions $\mathrm{T}, \theta, \theta^{(m)}$ are still well defined, and Lemmas 2.2 and 2.3 guarantee that $\theta^{(m)}(\mathbf{x}, \alpha)$ is a strictly increasing function of $\alpha$ for fixed $\mathbf{x}$ (but not necessarily continuous.) Hence, there is a unique value $\alpha_{0}$ such that

$$
\lim _{\alpha \uparrow \alpha_{0}} \theta^{(m)}(\mathbf{x}, \alpha) \leq 2 \pi \leq \lim _{\alpha \downarrow \alpha_{0}} \theta^{(m)}(\mathbf{x}, \alpha)
$$


for $m \geq 2$, where the upper limit inequality is omitted if $\alpha_{0}=2$. We claim that all equilateral $m$-gons have edge length $\alpha_{0}$. Indeed, the strict increasing property of $\theta^{(m)}(\mathbf{x}, \alpha)$ in $\alpha$ guarantees that any set of steps of edge length $>\alpha_{0}$ must overshoot rotation by $2 \pi$, while any step of edge length $<\alpha_{0}$ must undershoot rotation by $2 \pi$.

Lemma 2.4 allows us to assign unambiguously, for each point $x \in \partial B$, the edge length $\alpha_{m}(\mathbf{x} ; \mathbf{B})$ of an equilateral Minkowski $m$-gon having $\mathbf{x}$ as a vertex. The proof above shows that $\alpha_{2}(\mathbf{x}, \mathbf{B})=2$ in all cases. Lemma 2.3 yields

$$
2=\alpha_{2}(\mathbf{x}, \mathbf{B}) \geq \alpha_{3}(\mathbf{x}, \mathbf{B}) \geq \alpha_{4}(\mathbf{x}, \mathbf{B}) \geq \cdots
$$

Now define

$$
\alpha(m, \mathbf{B})=\sup \left\{\alpha_{m}(\mathbf{x}, \mathbf{B}): \mathbf{x} \in \partial \mathbf{B}\right\}
$$

which measures the edge length of the longest convex equilateral $m$-gon inscribable in $\partial \mathbf{B}$. The quantity $\alpha(m, \mathbf{B})$ is an affine invariant. Next define the extremal constants

$$
\begin{aligned}
& \alpha^{+}(m)=\sup \left\{\alpha_{m}(\mathbf{B}): \text { B a Minkowski disk }\right\} \\
& \alpha^{-}(m)=\inf \left\{\alpha_{m}(\mathbf{B}): \text { B a Minkowski disk }\right\} .
\end{aligned}
$$

It is well known that every Minkowski disk $\mathbf{B}$ has an affinely equivalent representative $\mathbf{B}^{\prime}=L(\mathbf{B})$ such that

$$
\mathbf{D} \subseteq \mathbf{B}^{\prime} \subseteq \sqrt{2} \mathbf{D}
$$

where $\mathbf{D}=\left\{\left(x_{1}, x_{2}\right): x_{1}^{2}+x_{2}^{2} \leq 1\right\}$ is the Euclidean disk. Using this fact a standard compactness argument allows us to show that there exist (not necessarily unique) extremal disks $\mathbf{B}_{m}^{+}$and $\mathbf{B}_{m}^{-}$attaining $\alpha^{+}(m)$ and $\alpha^{-}(m)$, respectively, for each $m \geq 3$. Thus we obtain:

Minkowski Equilateral $m$-gon Problems. Determine the extremal constants $\alpha^{+}(m)$, $\alpha^{-}(m)$ and corresponding extremal bodies $\mathbf{B}_{m}^{+}, \mathbf{B}_{m}^{-}$for each $m \geq 2$.

The case $m=6$ is settled by the following well-known result proved, for example, in [10].

Proposition 2.1. Let $\mathbf{B}$ be a Minkowski disk. Given any point $\mathbf{x}$ on the boundary of $\mathbf{B}$ there is an inscribed equilateral Minkowski hexagon of side length 1 having $\mathbf{x}$ as a vertex.

In combination with Lemma 2.4 this proposition gives $\alpha_{6}(\mathbf{x}, \mathbf{B})=1$ for all $\mathbf{x}$ 
and $\mathbf{B}$, hence the extremal constants for $m=6$ are

$$
\alpha^{+}(6)=\alpha^{-}(6)=1
$$

All Minkowski disks are extremal for $m=6$. Some other extremal constants are determined in Section 3.

Remark. There is an n-dimensional analogue of Lemma 2.3: When the unit ball $\mathbf{B}$ of an $n$-dimensional Minkowski space is smooth and strictly convex, the subset $\mathbf{S}$ of $\partial \mathbf{B}$ consisting of points at a constant distance $t(0<t<2)$ from some fixed point of $\partial \mathbf{B}$ is topologically an $(n-2)$-sphere. This is true because $\mathbf{S}=$ $\partial \mathbf{B} \cap\left(t \partial \mathbf{B}+\mathbf{x}_{0}\right)$, and it is a general fact that the boundaries of two homothetic copies of a smooth strictly convex body can only intersect in one of the four ways illustrated in Fig. 2.5, see [12]. To see that these are the only possibilities, note that as long as you are not in case (i), (ii), or (iii) then $\partial \mathbf{A}$ and $\partial \mathbf{B}$ must intersect transversally, because the tangent spaces at two different boundary points of a smooth strictly convex body can coincide only for antipodal points. Thus the intersection $\mathbf{S}$ is some smooth manifold. That it is an $(n-2)$-sphere is clear if $\mathbf{B}$ is much smaller than $\mathbf{A}$ and if the center of $\mathbf{B}$ is located on $\partial \mathbf{A}$, since in this case the intersection will approximate the intersection of $\partial \mathbf{B}$ with a hyperplane. However, we can always smoothly homotope the picture to this situation by varying $t$ and $x_{0}$ without ever encountering case (i), (ii), or (iii). The intersection remains transversal throughout this homotopy, so no surgeries are taking place, and the intersection must remain topologically the same throughout the homotopy, i.e., it must be an $(n-2)$-sphere. In the case relevant to Lemma 2.3 , the intersection is a 0 -sphere-that is, two points-and it evolves as pictured in Fig. 2.6.

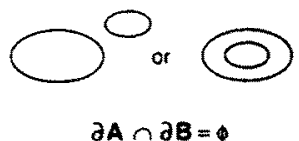

(i)

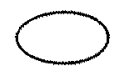

$\mathbf{A}=\mathbf{B}$. and $\partial A \cap \partial B=S^{n-1}$

(iii)

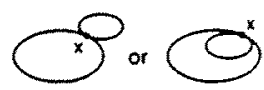

$\partial A \cap \partial B=\{x\}$

(ii)

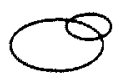

$\partial A$ and $\partial B$ are transversal
and $\partial A \cap \partial B=S^{n-2}$

(iv)

Fig. 2.5. Boundaries of two homothetic copies of a smooth, strictly convex body in $\mathbb{R}^{n}$. 


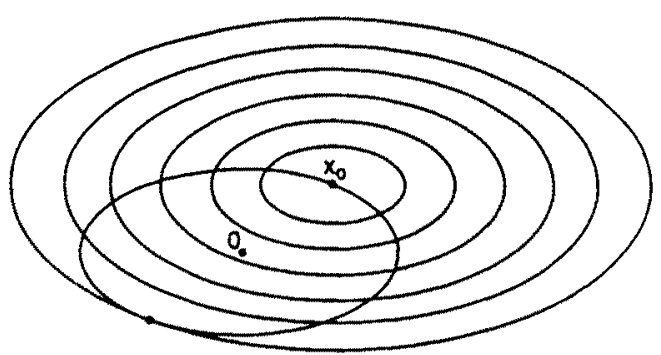

Fig. 2.6. $\left(t \partial \mathbf{B}+x_{\mathrm{o}}\right) \cap \partial \mathbf{B}$ for $0 \leq t \leq 2$

\section{Self-Packing Constants and Equilateral Polygons}

Self-packing constants $\rho(m, \mathbf{B})$ and equilateral $m$-gon lengths $\alpha(m, \mathbf{B})$ are related for small values of $m$, because there exist extremal packing configurations having polygonal shape.

Theorem 3.1. For all Minkowski disks B and all $m \leq 6$,

$$
\rho(m, \mathbf{B})=1+\frac{2}{\alpha(m, \mathbf{B})} .
$$

Proof. We have, for all $m \geq 2$,

$$
\rho(m, \mathbf{B}) \leq 1+\frac{2}{\alpha(m, \mathbf{B})} .
$$

Indeed, by the definition of $\alpha(m, \mathbf{B})$ we can find $m$ points $\left\{\mathbf{x}_{i}\right\}$ on $\partial \mathbf{B}$ forming a convex polygon of Minkowski side-length $\alpha(m, B)$, and using Lemma 2.3 the Minkowski distance between each pair of points is at least $\alpha(m, \mathbf{B})$. Hence, we can place open Minkowski disks of radius $\frac{1}{2} \alpha(m, B)$ at these points without overlap. Each of these disks $x_{i}+\frac{1}{2} \alpha(m, B) B$ is contained in the Minkowski disk of radius $1+\frac{1}{2} \alpha(m, \mathbf{B})$ centered at $\mathbf{0}$, since

$$
d(\mathbf{y}, \mathbf{0}) \leq d\left(\mathbf{0}, \mathbf{x}_{i}\right)+d\left(\mathbf{x}_{i}, \mathbf{y}\right) \leq 1+\frac{1}{2} \alpha(m, \mathbf{B})
$$

Now homothetically enlarge this configuration by a factor of $\left(\frac{1}{2} \alpha(m, \mathbf{B})\right)^{-1}$ and (3.2) follows.

It remains to show for $m \leq 6$ the lower bound

$$
\rho(m, \mathbf{B}) \geq 1+\frac{2}{\alpha(m, \mathbf{B})} .
$$

We know from Section 2 that, for $m \leq 6$,

$$
\alpha(m, \mathbf{B}) \geq \alpha(6, \mathbf{B})=1,
$$


hence, the upper bound (3.2) yields $\rho(m, \mathbf{B}) \leq 3$. If $\rho(m, \mathbf{B})=3$, then necessarily $\alpha(m, B)=1$, and (3.2) and (3.3) hold with equality.

It remains to establish $(3.3)$ in the case that $\rho(m, \mathbf{B})<3$. We first prove this for strictly convex bodies $\mathbf{B}$ and then settle the general case by a continuity argument. Suppose that we are given an extremal configuration of $m$ bodies $\left\{\mathbf{w}_{i}+\mathbf{B}: 1 \leq i \leq m\right\}$ packed inside $\rho \mathbf{B}$ where $\rho=\rho(m, \mathbf{B})<3$. Our approach is to show that we can move the centers $\mathbf{w}_{i}$ to form an equilateral Minkowski $m$-gon inscribed in $(\rho-1) \mathbf{B}$ that still packs inside $\rho \mathbf{B}$. It proves convenient to scale the configuration homothetically by a factor $\beta=1 /(\rho-1)$ so that we have $m$ Minkowski disks of radius $\beta>\frac{1}{2}$ packed in a disk of radius $\rho /(\rho-1)=1+\beta$. The resulting centers $\left\{\mathbf{x}_{i}: 1 \leq i \leq m\right\}$ of the $m$ scaled disks now have

$$
d\left(\mathbf{x}_{i}, \mathbf{x}_{j}\right) \geq 2 \beta>1 \text {. }
$$

Next we claim that all $\mathbf{x}_{i}$ lie in $\mathbf{B}$, i.e.,

$$
d\left(\mathbf{x}_{i}, \mathbf{0}\right) \leq 1
$$

For if not, extending a ray in the direction $\mathbf{x}_{i}$ inside $x_{i}+\beta \mathbf{B}$ for Minkowski length $\beta$ we obtain a point $(1+u) \mathbf{x}_{i}$ in this disk with

$$
d\left(\mathbf{0},(1+\mu) \mathbf{x}_{i}\right)=d\left(\mathbf{x}_{i}, \mathbf{0}\right)+\beta>1+\beta,
$$

contradicting the hypothesis that all points lie in $(1+\beta) \mathbf{B}$. Observe also that no $\mathbf{x}_{i}=\mathbf{0}$. For if some $\mathbf{x}_{i}=0$, then (3.4) would contradict (3.5).

Now create a new configuration $\left\{\mathbf{y}_{i}: 1 \leq i \leq n\right\}$ by radially scaling the $\mathbf{x}_{i}$ to lie on $\partial \mathbf{B}$, i.e.,

$$
\mathbf{y}_{i}=d\left(\mathbf{x}_{i}, 0\right)^{-1} \mathbf{x}_{i}
$$

This can always be done since all $\mathbf{x}_{i} \neq \mathbf{0}$. Now the hypotheses of Lemma 2.3 are satisfied, and in consequence we have

$$
d\left(\mathbf{y}_{i}, \mathbf{y}_{j}\right) \geq d\left(\mathbf{x}_{i}, \mathbf{x}_{j}\right) \geq 2 \beta_{1}>1
$$

Next we claim that there exists a configuration $\left\{\mathbf{z}_{i}\right\}$ in $\partial \mathbf{B}$ such that:

(1) $\left\{\mathbf{z}_{i}\right\}$ form a convex equilateral Minkowski $m$-gon.

(2) $\min _{i \neq j}\left[d\left(\mathbf{z}_{i}, \mathbf{z}_{j}\right)\right] \geq \min _{i \neq j}\left[d\left(\mathbf{y}_{i}, \mathbf{y}_{j}\right)\right]$.

To see this, relabel the $\mathbf{y}_{i}$ counterclockwise around the boundary $\partial \mathbf{B}$ as $\mathbf{y}_{1}$, $\mathbf{y}_{2}, \ldots, \mathbf{y}_{m}$. Now set $\gamma=\min _{i \neq j}\left[d\left(\mathbf{y}_{i}, \mathbf{y}_{j}\right)\right]>1$ and compare the angular locations measured from $\mathbf{y}_{m}$ of $T_{y}^{(k)}\left(\mathbf{y}_{m}\right)$ with $\mathbf{y}_{k}$ for $1 \leq k \leq m$. If $\varphi_{k}$ denotes the change in argument of $\mathbf{y}_{k}$ measured counterclockwise starting from $\mathbf{y}_{m}$, then by Lemma 2.3 we show inductively that

$$
\varphi_{k} \geq \theta^{(k)}\left(\mathbf{y}_{m}, \gamma\right), \quad 1 \leq k \leq m,
$$


because the map $T_{\gamma}$ takes a step of length $\gamma$ and the step from $y_{k-1}$ to $y_{k}$ is always of length $\geq \gamma$, so that $T^{(k)}\left(\mathbf{y}_{m}\right)$ always lags behind $\mathbf{y}_{k}$ and can never pass it. Consequently,

$$
\theta^{(m)}\left(\mathbf{y}_{m}, \gamma\right) \leq 2 \pi
$$

Now we take $\gamma_{1}$ with $\theta^{(m)}\left(\mathbf{y}_{m}, \gamma_{1}\right)=2 \pi$ and set

$$
\mathbf{z}_{k}=T_{\gamma_{1}}^{(k)}\left(\mathbf{y}_{m}\right), \quad 1 \leq k \leq m,
$$

which forms an equilateral Minkowski $m$-gon on $\partial \mathbf{B}$ of side length $\gamma_{1} \geq \gamma$, proving the claim.

The claim shows that

$$
\alpha(m, \mathbf{B}) \geq \gamma_{1} \geq \gamma \geq 2 \beta=\frac{2}{\rho-1},
$$

and this inequality is equivalent to (3.3).

For the case of general B, it suffices to observe that $\alpha(m, \mathbf{B})$ and $\rho(m, \mathbf{B})$ will both be continuous functions in any sensible metric on the set of affine equivalence classes of symmetric convex bodies, see p. 235 of [9].

Remark. John H. Conway claims that if you can pack six pennies in a disk, then you can slide them around inside without picking them up or letting them overlap during the sliding so that when you are all done each penny touches the boundary of the disk. As applied to pennies the result above is weaker: it merely shows that the disk is big enough so that you can pick the pennies up and then pack them back in so that each penny touches the boundary of the disk.

The proof above actually determines all the extremal packings for strictly convex B. Examination of the conditions for equality in (3.6) shows that: For a strictly convex Minkowski disk B and all $m \leq 6$, all extremal configurations $\left\{\mathbf{y}_{i}+\mathbf{B}: 1 \leq i \leq m\right\}$ must have $\mathbf{y}_{i}$ forming a maximal perimeter equilateral Minkowski m-gon inscribed in $(\rho-1) \mathbf{B}$.

We immediately deduce from this result the self-packing radius for six or seven bodies.

Theorem 3.2. For all Minkowski disks B we have

$$
\rho(6, \mathrm{~B})=\rho(7, \mathrm{~B})=3 .
$$

Proof. Theorem 3.1 and the result $\alpha(6, \mathrm{~B})=1$ of Proposition 2.1 yield $\rho(6, \mathrm{~B})=3$.

However, there is always a packing of seven bodies inside $3 \mathbf{B}$, because when six bodies are put at the vertices of an equilateral hexagon of Minkowski side length 2 on the boundary of $2 B$, then a seventh copy of $B$ fits in when centered at $\mathbf{0}$. Thus $\rho(6, \mathbf{B}) \leq \rho(7, \mathbf{B}) \leq 3$, so we are done. 


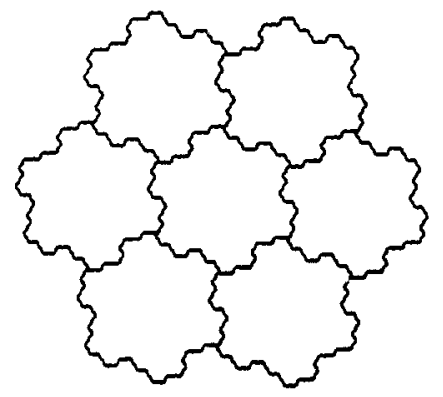

Fig. 3.1. Self-packing for fractal tile F.

It is immediate that for all $\mathbf{B}$ we have

$$
\rho(2, \mathbf{B})=2 \text {. }
$$

It appears that $m=2,6$, and 7 are the only values of $m$ for which $\rho(m, \mathbf{B})$ is independent of the shape of $\mathbf{B}$. We easily show that $\rho(m, \mathbf{B})$ depends on $\mathbf{B}$ for $m=3,4$, and 5 using Theorem 3.1, and this also holds for all $m \geq 3600$ using Theorem 4.1 below; we have not ruled out the remaining $m$ though this does not seem hard.

The convexity and central-symmetry of $\mathbf{K}$ play an essential role in Theorem 3.2 , for there exists a nonconvex body $\mathbf{F}$ which is the closure of its interior and has the property that seven translates of $\mathbf{F}$ perfectly pack $\sqrt{7} \mathbf{F}$, so that $\rho(7, F)=\sqrt{7}$. The body $\mathbf{F}$ is pictured in Fig. 3.1. It has a fractal boundary of Hausdorff dimension $(\log 3) /(\log \sqrt{7})=1.12915 \ldots$ The body $F$ appears on p. 46 of [14], where its construction is credited to W. Gosper, see also [22].

Now define the extremal constants

$$
\begin{aligned}
& \rho^{+}(m)=\sup \{\rho(m, \mathbf{B}): \mathbf{B} \text { a Minkowski disk }\} \\
& \rho^{-}(m)=\inf \{\rho(m, \mathbf{B}): \text { B a Minkowski disk }\}
\end{aligned}
$$

Theorem 3.1 implies for $m \leq 6$ that

$$
\begin{aligned}
& \rho^{+}(m)=1+\frac{2}{\alpha^{-}(m)}, \\
& \rho^{-}(m)=1+\frac{2}{\alpha^{+}(m)} .
\end{aligned}
$$

The square $\mathbf{S}$ is an extremal body for many values of $m$. We clearly have

$$
\rho(m, \mathbf{S})=k+1 \quad \text { for } \quad k^{2}<m \leq(k+1)^{2} \text {. }
$$


It provides several small extremal constants:

$$
\begin{array}{ll}
\rho^{-}(3)=2, & \alpha^{+}(3)=2, \\
\rho^{-}(4)=2, & \alpha^{+}(4)=2, \\
\rho^{+}(5)=3, & \alpha^{-}(5)=1,
\end{array}
$$

which follow on observing that, for $3 \leq m \leq 5,3=\rho^{+}(6) \geq \rho^{+}(m) \geq \rho^{-}(m) \geq$ $\rho^{-}(2)=2$.

We determine one other extremal constant.

Theorem 3.3. The circular disk is the hardest body to self-pack with four copies of itself. We have

$$
\rho^{+}(4)=1+\sqrt{2}, \quad \alpha^{-}(4)=\sqrt{2} \text {. }
$$

Proof. By Theorem 3.1 it suffices to show that any Minkowski disk B contains an equilateral 4-gon of side length at least $\sqrt{2}$. Now an equilateral Minkowski 4-gon is a parallelogram. Take an extremal 4-gon for $\mathbf{B}$ and without loss of generality we may suppose, by making a suitable affine transformation, that it is a square $\mathbf{S}$ with sides parallel to the axes, of Euclidean length 1. This can be done since $\alpha(m, B)$ is unchanged by affine transformations, see Fig. 3.2. Now the Minkowski length of a vertical side of $\mathbf{S}$ is $2 / r_{1}$ where $r_{1}$ is the Euclidean length of the vertical diameter of $\mathbf{B}$ passing through $\mathbf{0}$, and that of a horizontal side is $2 / r_{2}$ where $r_{2}$ is the length of the horizontal diameter in $\mathbf{B}$ passing through $\mathbf{0}$. Since this square is Minkowski equilateral, $r_{1}=r_{2}=r$. Now consider the square of side $r / \sqrt{2}$ passing through the four endpoints of these two segments, which has sides at angles $\pm \pi / 4$ to the axes. It is inscribed in $\partial \mathbf{B}$, and the Minkowski length of each side of this square is exactly $r$, because the four corners of the original square are on $\partial \mathbf{B}$ at Euclidean distance $1 / \sqrt{2}$ from the origin and the segments connecting them to $\mathbf{0}$ are parallel to the sides of the new square. Hence we have two equilateral Minkowski 4-gons inscribed in $\partial \mathbf{B}$ with Minkowski side lengths $r$ and $2 / r$, respectively. Consequently

$$
\alpha(4, B) \geq \max \left(r, \frac{2}{r}\right) \geq \sqrt{2} .
$$

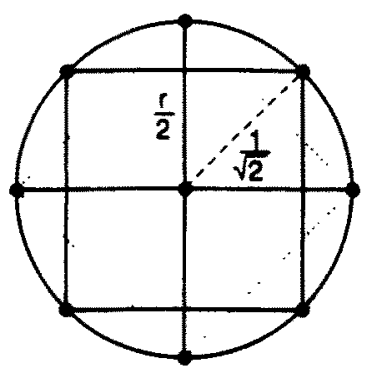

Fig. 3.2. Equilateral 4-gons in a Minkowski disk. 
However, the Euclidean disk $D$ has $\alpha(4, D)=\sqrt{2}$, hence $\alpha^{-}(4)=2$ and the theorem follows.

We have thus determined all the extremal self-packing constants $\rho^{ \pm}(m)$ for $m \leq 7$ except for $\rho^{+}(3)$ and $\rho^{-}(5)$. The regular octagon $\mathbf{P}_{8}$ gives the largest value of $\rho(3, \mathbf{B})$ that we have found. It has the property that all inscribed equilateral triangles have the same side length $1+1 / \sqrt{2}$, hence $\rho\left(3, \mathbf{P}_{8}\right)=5-2 \sqrt{2} \doteq 2.172$, using Theorem 3.1 .

The quantity $\rho^{+}(3)$ gives some information about the extremal ratio of Steiner minimal trees to minimal spanning trees for Minkowski spaces.

Theorem 3.4. For any Minkowski space in $\mathbb{R}^{2}$ there exists a configuration of three points such that

$$
\frac{S T}{M S T} \leq \frac{3}{2 \alpha^{-}(3)}=\frac{3}{4}\left(\rho^{+}(3)-1\right),
$$

where ST is the Steiner minimal tree and MST is the minimal spanning tree for this configuration.

Proof. Given a Minkowski disk, take three points determining the inscribed equilateral triangle of largest edge length, which is at least $\alpha^{-}$(3). The bound follows on choosing to add the origin as a Steiner point.

This bound gives something away because the origin need not give a minimal Steiner tree. Du et al. [3] conjecture that $\sqrt{3} / 2 \doteq 0.8660$ is the best possible bound. The regular octagon example shows that the right-hand side of (3.9) must be at least as large as $3 /(2+\sqrt{2}) \doteq 0.8787$.

\section{Self-Packing Radii for Arbitrary $m$}

The asymptotic behavior of self-packing radii $\rho(m, \mathbf{B})$ as $m \rightarrow \infty$ obviously depends on the packing density attainable by the body. The packing density $\delta(\mathbf{B})$ is that percentage of $\mathbb{R}^{2}$ that can be covered by a densest packing of translates of $\mathbf{B}$, defined as a limit of packing density of a packing in a square of side $t \rightarrow \infty$, see [20] for a precise definition. Fejes-Tóth [6] and Rogers [19] proved for centrally symmetric B that

$$
\delta(\mathbf{B})=\frac{\operatorname{Vol}(\mathbf{B})}{\operatorname{Vol}(\mathbf{H})}
$$

where $\mathbf{H}$ is the minimum volume hexagon containing $\mathbf{B}$, and this hexagon $\mathbf{H}$ can always be chosen to be centrally symmetric and convex.

The following result gives a bound for the self-packing radius that is valid for 
all $m \geq 2$. It uses an inequality of Oler [16] for a slackness function which was introduced by Zassenhaus as an aid in studying the tightness of finite packings, see [24].

Theorem 4.1. For all Minkowski disks $\mathbf{B}$ in $\mathbb{R}^{2}$ and all $m \geq 2$ we have

$$
\left(\frac{m}{\delta(\mathbf{B})}\right)^{1 / 2}-\frac{3}{2} \leq \rho(m, \mathbf{B}) \leq\left(\frac{m}{\delta(\mathbf{B})}\right)^{1 / 2}+1
$$

Proof. We study the inverse function $\eta(t, \mathbf{B})$ which counts the maximal number of translates of $\mathbf{B}$ that are packable into $t \mathbf{B}$. Let $\mathbf{J}$ denote the convex hull of the centers of such a packing of $t \mathbf{B}$. We use the main inequality of Oler [16], a special case of which gives

$$
\delta(\mathbf{B}) \frac{\operatorname{Area}(\mathbf{J})}{\operatorname{Area}(\mathbf{B})}+\frac{1}{2} P(\boldsymbol{J})+1 \geq \eta(t, \mathbf{B}),
$$

where $P(J)$ is the Minkowski length of the perimeter of $J$. To simplify this inequality, we use the bound

$$
\operatorname{Area}(\mathbf{J}) \leq \operatorname{Area}((t-1) \mathbf{B})=(t-1)^{2} \operatorname{Area}(\mathbf{B})
$$

which holds since all vertices of $\mathbf{J}$ are at Minkowski distance at least 1 inside $t \mathbf{B}$.

We also need the following fact: For convex bodies $\mathbf{J}_{1} \subseteq \mathbf{J}_{2}$ we have $P\left(\mathrm{~J}_{1}\right) \leq P\left(\mathrm{~J}_{2}\right)$. To prove this we may use the observation due to Minkowski $[15$, p. 462] (see also p. 864 of [2]) that the Minkowski perimeter of a body $K$ with respect to the metric of $B$ is expressible as a mixed volume

$$
P(\mathbf{K})=2 V\left(\mathbf{K}, \mathbf{B}^{*}\right)
$$

where $\mathrm{B}^{*}$ is the polar reciprocal body of $\mathbf{B}$ rotated by $\pi / 4$. Minkowski proved monotonicity of all mixed volumes under inclusion (see p. 41 of [1]), which as a special case for $\mathbf{J}_{1} \subseteq \mathbf{J}_{2}$ gives $V\left(\mathbf{J}_{1}, \mathbf{B}^{*}\right) \subseteq V\left(\mathbf{J}_{2}, \mathbf{B}^{*}\right)$ and the fact follows.

Now since $\mathbf{J} \subseteq t \mathbf{B}$ the fact gives

$$
P(\mathbf{J}) \leq P(t \mathbf{B})=t P(\mathbf{B}) \leq 8 t,
$$

using the result that the self-perimeter $P(\mathbf{B}) \leq 8$ for all Minkowski disks (see p. 35 of [21]). Substituting (4.4) and (4.5) in (4.3) yields

$$
(t-1)^{2} \delta(\mathbf{B})+4 t+1 \geq \eta(t, \mathbf{B})
$$

To obtain a lower bound for $\eta(t, \mathbf{B})$, take a minimal volume circumscribing hexagon $\mathbf{H}$ that is convex and centrally symmetric. Then $\mathbf{H}$ tiles the plane with a 
lattice tiling. Now take the configuration consisting of all centers of this lattice that lie in $(t-1) \mathbf{B}$, which produces an admissible packing in $t \mathbf{B}$, consisting of $N$ disks, say. Let $\mathbf{K}$ denote the set of hexagons $\mathbf{H}$ centered at these $N$ points, and observe that $(t-1) \mathbf{B} \subseteq \mathbf{K}$, so that

$$
N \geq \frac{\operatorname{vol}((t-1) \mathbf{B})}{\operatorname{vol}(\mathbf{H})} \geq(t-1)^{2} \delta(\mathbf{B}) .
$$

Since $\eta(t, \mathbf{B}) \geq N$ we get the lower bound

$$
\eta(t, \mathbf{B}) \geq(t-1)^{2} \delta(\mathbf{B})
$$

Now we have

$$
\alpha(m, \mathbf{B})=\inf \{t: \eta(t, \mathbf{B}) \geq m\} .
$$

Then (4.7) immediately gives the upper bound

$$
\alpha(m, \mathbf{B}) \leq\left(\frac{m}{\delta(\mathbf{B})}\right)^{1 / 2}+1 .
$$

For the lower bound, we observe that Mahler [13] and Fejes Tóth [5] showed that

$$
\delta(\mathbf{B}) \geq \frac{\sqrt{3}}{2},
$$

see also [11]. Hence $\delta(\mathbf{B}) \geq \frac{4}{5}$ and (4.6) implies that

$$
\left(t+\frac{3}{2}\right)^{2} \delta(\mathbf{B}) \geq \eta(t, \mathbf{B}),
$$

which immediately gives the lower bound

$$
\alpha(m, \mathbf{B}) \geq\left(\frac{m}{\delta(\mathbf{B})}\right)^{1 / 2}-\frac{3}{2} .
$$

The example (3.8) of the square $S$ shows that the upper bound in (4.2) is asymptotically sharp. The constant in the lower bound can be improved for large enough $m$ to -1.24 using the bound $\delta(\mathbf{B}) \geq 0.89265$ of Tammela [23], but it seems a hard problem to determine the asymptotically best constant as $m \rightarrow \infty$. The example of the square shows that the lower bound constant can at most be increased to 0 , as $m \rightarrow \infty$.

Further results about slackness function inequalities can be found in [17] and [7].

Concerning the packing constant $\delta(\mathbf{B})$, it is well known that $\delta(\mathbf{B}) \leq 1$ with 
equality attained only for $\mathbf{B}$ being a parallelogram or a centrally symmetric convex hexagon. The body $\mathbf{B}$ with the smallest known packing constant $\delta(\mathbf{B}) \cong 0.901$ is a regular octagon with corners rounded off by hyperbolic arcs. It was discovered by Reinhardt [18], who conjectured it is extremal. This has never been proved.

Finally, we remark that the question of which convex bodies allow perfect self-packings was settled by Groemer [8, Hilfssatz 2]. He showed that if $\mathbf{K}$ is a convex body in $\mathbb{R}^{n}$, copies of which can perfectly pack some larger homothetic copy $t \mathbf{K}$ of itself, then $\mathbf{K}$ is a parallelepiped. The body pictured in Fig. 3.1 shows that the assumption that $\mathbf{K}$ is convex is necessary in Groemer's result.

\section{Acknowledgment}

We are indebted to the referees for pointing out a slip in our original proof of Theorem 4.1.

\section{References}

1. T. Bonnesen and W. Fenchel, Theorie der konvexen Körper, Springer-Verlag, Berlin, 1934. (Translation: Theory of Convex Bodies, BCS Associates, Moscow, 1987,)

2. H. Busemann, The isoperimetric inequality for the Minkowski plane, Amer. J. Math. 69 (1947), 863-871.

3. D.-Z. Du, R. L. Graham, and Z. C. Liu, Minimal Steiner Trees in Normed Planes, preprint, 1991.

4. D.-Z. Du and F. K. Hwang, The Steiner-ratio conjecture of Gilbert-Pollak is true, Proc. Nat. Acad. Sci. USA 87 (1990), 9464-9466.

5. L. Fejes Tóth, On the densest packing of domains, Proc. Kon. Ned. Acad. Wet. S1 (1948), 189-192.

6. L. Fejes Tóth, Some packing and covering theorems, Acta. Sci. Math. (Szeged) 12/A (1950), 62-67.

7. R. L. Graham, H. S. Witsenhausen, and H. Zassenhaus, On tightest packings in the Minkowski plane, Pacific J. Math. 41 (1972), 699-715.

8. H. Groemer, Abschätzungen für die Anzahl der konvexen Körper, die einen konvexen Körper berühren, Monatsh. Math. 65 (1961), 74-81.

9. B. Grünbaum, Measures of symmetry for convex sets, in Convexity, pp. 233-270, Proc. Sympos. Pure Math. No. 7, American Mathematical Society, Providence, RI, 1963.

10. P. J. Kelly, A property of Minkowski circles, Amer. Math. Monthly 57 (1950), 677-678.

11. G. Kuperberg and W. Kuperberg, Double lattice packings of convex bodies in the plane, Discrete Comput. Geom. 5 (1990), 389-397.

12. G. Lawlor and F. Morgan, Paired calibrations applied to soap films, immiscible fluids, and surfaces and networks minimizing other norms, preprint, 1991.

13. K. Mahler, The theorem of Minkowski-Hlawka, Duke Math. J, 13 (1946), 611-621.

14. B. B. Mandelbrot, The Fractal Geometry of Nature, Freeman, New York, 1977.

15. H. Minkowski, Volumen und Oberflache, Math. Ann. 57 (1903), 447-593. (Gesammelte Abhandlungen von Hermann Minkowski, Vol. II, Chelsea, New York, pp. 230-278.)

16. N. Oler, An inequality in the geometry of numbers, Acta Math. 105 (1961), 19-48.

17. N. Oler, The slackness of finite packings in $\mathbb{R}^{2}$, Amer. Math. Monthly 69 (1964), 511-514.

18. K. Reinhardt, Über die dichteste gitterförmige Lagerung kongruenter Bereiche in der Ebene und eine besondere Art convexer Kurven, Abh. Math. Sem. Univ. Hamburg 10 (1934), 216-230.

19. C. A. Rogers, The closest packing of convex two-dimensional domains, Acta. Math. 86 (1951), 309-321. (Corrigendum: 104 (1961), 305-306.)

20. C. A. Rogers, Packing and Covering, Cambridge University Press, Cambridge, 1964.

21. H. Rund, The Differential Geometry of Finsler Spaces, Springer-Verlag, Berlin, 1959. 
22. M. Schroeder, Fractals, Chaos, Power-Laws: Minutes from an Infinite Paradise, Freeman, San Francisco, 1991.

23. P. Tammela, An estimate of the critical determinant of a two-dimensional convex symmetric domain, Izv. Vyssh. Uchebn. Zaved. Mat. 12 (1970), 103-107 (in Russian).

24. H. Zassenhaus, Modern developments in the geometry of numbers, Bull. Amer. Math. Soc. 67 (1961), $427-439$.

Received December 10,1990, and in revised form April 19, 1991, July 8,1991 and November 10, 1991. 